\title{
Factors associated with mode of colorectal cancer detection and time to diagnosis: a population level study
}

\author{
Khokan C. Sikdar ${ }^{*}$, James Dickinson ${ }^{2}$ and Marcy Winget ${ }^{3}$
}

\begin{abstract}
Background: Although it is well-known that early detection of colorectal cancer (CRC) is important for optimal patient survival, the relationship of patient and health system factors with delayed diagnosis are unclear. The purpose of this study was to identify the demographic, clinical and healthcare factors related to mode of CRC detection and length of the diagnostic interval.

Methods: All residents of Alberta, Canada diagnosed with first-ever incident CRC in years 2004-2010 were identified from the Alberta Cancer Registry. Population-based administrative health datasets, including hospital discharge abstract, ambulatory care classification system and physician billing data, were used to identify healthcare services related to CRC diagnosis. The time to diagnosis was defined as the time from the first CRC-related healthcare visit to the date of CRC diagnosis. Mode of CRC detection was classified into three groups: urgent, screen-detected and symptomatic. Quantile regression was performed to assess factors associated with time to diagnosis.
\end{abstract}

Results: 9626 patients were included in the study; 25\% of patients presented as urgent, 32\% were screen-detected and $43 \%$ were symptomatic. The median time to diagnosis for urgent, screen-detected and symptomatic patients were 6 days (interquartile range (IQR) 2-14 days), 74 days (IQR 36-183 days), 84 days (IQR 39-223 days), respectively. Time to diagnosis was greater than 6 months for $27 \%$ of non-urgent patients. Healthcare factors had the largest impact on time to diagnosis: 3 or more visits to a GP increased the median by 140 days whereas 2 or more visits to a Gl-specialist increased it by 108 days compared to $0-1$ visits to a GP or Gl-specialist, respectively.

Conclusion: A large proportion of CRC patients required urgent work-up or had to wait more than 6 months for diagnosis. Actions are needed to reduce the frequency of urgent presentation as well as improve the timeliness of diagnosis. Findings suggest a need to improve coordination of care across multiple providers.

Keywords: Colorectal cancer, Diagnostic delay, Wait time, Primary health care, Administrative data

\section{Background}

Colorectal cancer (CRC) is the second most common cancer in men and the third most common cancer in women in Canada. Every year almost 24,000 Canadians are diagnosed with and 9,200 die from CRC [1]. The diagnosis of CRC is often not straightforward. Symptoms are often non-specific and commonly occur in benign conditions [2]. Presentation and diagnosis are therefore often delayed until the cancer is advanced [3]. Screening

\footnotetext{
* Correspondence: khokan.sikdar@ucalgary.ca

${ }^{1}$ Community Health Sciences and O'Brien Institute for Public Health, Cumming School of Medicine, University of Calgary, G 214 HSC, 3330 Hospital Drive NW, Calgary, AB T2N 4N1, Canada

Full list of author information is available at the end of the article
}

asymptomatic individuals can effectively detect CRC earlier; however, many nations, including Canada, have either a relatively new or no colorectal screening program $[4,5]$. Early diagnosis of CRC is important because 5 -year survival is much higher $(90 \%)$ than that from metastatic disease (11\%) [6].

A long time from symptom onset to diagnosis or treatment has been shown to contribute to cancer progression, higher stage disease and worse survival for cancer patients [7-10]. Individuals diagnosed promptly may benefit from timely treatment, which would improve prognosis and decrease patient anxiety [11]. 
Although there is consensus that patients should be diagnosed in a timely manner, there is little information regarding a reasonable length of diagnostic interval, that is, time from patient first presentation to the healthcare system to definitive diagnosis, or understanding of what factors affect the length of the diagnostic interval. Whether patients are diagnosed via screening or symptomatic presentation, a typical pre-diagnostic work-up may include visits to a family physician/general practitioner (GP), diagnostic imaging tests, follow-up visits and endoscopy, requiring a healthcare contacts with a variety of care providers.

The patients who present with advanced stage cancer are likely to be sicker, receive urgent care and be diagnosed more quickly than those who initially present with early stage cancer [10]. Some in this latter group may experience a prolonged diagnostic process because their symptoms are not severe or specific, or they have a chronic gastrointestinal (GI) condition that complicates diagnosing the cancer. Others that are asymptomatic may be diagnosed through screening. Screening may be conducted through a formal program or via opportunistic screening, as part of a visit for other reasons, notably for preventive care. Furthermore, the diagnostic work-up for CRC differs from most other common cancers because most CRCs are diagnosed by lower gastrointestinal endoscopy [12]. Given the complexity of the relationship between the patient's clinical condition at first presentation, health system factors and the diagnostic interval, investigation is warranted to better understand the relationship between these factors.

The purpose of the present study was to: 1) estimate the proportion of CRC patients that are detected via an opportunistic screening visit, urgent and non-urgent symptom visits; 2 ) identify patient demographic, clinical and healthcare utilization factors related to mode of CRC detection; and 3) identify patient demographic, clinical and healthcare utilization factors related to time to diagnosis for non-urgent cases.

\section{Methods}

\section{Study population}

All residents of Alberta, Canada diagnosed in years 2004 to 2010 with malignant, invasive CRC (International Classification of Disease for Oncology (ICD-O) $3^{\text {rd }}$ edition codes $\mathrm{C} 18-\mathrm{C} 20$ ) were identified from the Alberta Cancer Registry. Individuals with a first-ever primary colorectal cancer were included. Patients were excluded for the following reasons: 1) stage 0 cancer; 2) histology that is not of colon origin (e.g., sarcomas, melanomas, lymphomas); 3) unknown stage; 4) disease was not pathologically or microscopically confirmed; 5) prior history of CRC or stomach cancer; 6) diagnosis of another cancer within 6 months prior to diagnosis of CRC; and 7) missing data that prevented identification of first visit prompting diagnostic work-up.

\section{Data sources and relevant healthcare encounters}

Additional information obtained from the cancer registry included age and residence at diagnosis, anatomic site, and stage and date of diagnosis of tumor. The quality and completeness of the Alberta Cancer Registry has consistently been shown to be very high [13].

Health service utilization prior to and related to CRC diagnosis was obtained from three provincial administrative health databases: 1) the Discharge Abstract Database that contains inpatient data from all Alberta hospitals; 2) the Ambulatory Care Classification System Database that contains outpatient data from all Alberta hospitals; and 3) the Physician Billing Database that contains billing claims submitted by all physicians in Alberta remunerated on a fee-for-service basis. These three population-based datasets are routinely used in Canada to conduct health services research. Validation studies have shown them to have excellent sensitivity and specificity for identifying colorectal-related procedures and have high face validity for diagnosis codes [14-16]. Health services related to CRC diagnosis were defined using both procedure and diagnosis codes. Visits with procedure codes of: 1) diagnostic imaging to the abdominal or GI region including computerized tomography, ultrasound, X-ray or barium enema, or 2) lower endoscopy including colonoscopy and sigmoidoscopy were defined as related to CRC diagnosis. Physician visits with an ICD-9 or ICD-10 diagnosis code for any of: rectal bleeding, anaemia, abdominal pain, obstruction, abscess/ fistula, constipation, perforation, diarrhea, weight loss, or change in bowel habit were defined as CRC-related [17-19]. Visits with any of the above diagnostic codes were defined as visits with CRC-relevant symptoms. 'Symptom visits' that occurred in the absence of a CRCrelated procedure were further classified by type of physician as follows: 1) 'GI specialist' included gastroenterology, internal medicine and general surgery; 2) 'non-GI specialist' included all other specialists; 3) 'GP' included general practitioners, primary care and family physicians; and 4) 'unknown provider-type' when provider code was missing.

Diagnosis codes were also used to calculate Charlson co-morbidity scores using ICD-9/10 codes in the year prior to colorectal cancer diagnosis as described by Deyo et al. and updated for ICD-10 codes developed by Quan et al. $[20,21]$. The Charlson comorbidity index was originally developed to estimate death within one year of hospitalization. The Charlson-Deyo index considers 17 comorbid conditions each with an associated weight, ranging from 1 to 6 , based on the adjusted risk of mortality. The sum of all the weights results in a single 
comorbidity score for a patient. The Charlson score for each patient was categorized into three groups: 0,1 and $\geq 2$ to indicate no, mild and moderate-to-severe comorbidities. Chronic gastrointestinal (GI) tract conditions were also identified using ICD-9/10 codes in the year prior to diagnosis including: irritable bowel syndrome, irritable colon, Crohn's disease, ulcerative colitis, enteritis and diverticular disease of intestine, ulcer of anus and rectum and chronic vascular insufficiency intestine. These conditions are distinct from the Charlson comorbidities as they were selected based on discussion with the authors and additional primary care physicians regarding conditions that may complicate the diagnosis of CRC, as opposed to predicting mortality. Each patient was identified with presence or absence of GI-related comorbidities. The Usual Provider Continuity (UPC) index, a measure of continuity of care with a primary care physician, was derived based on GP visits in the two years prior to diagnosis [22]. Statistics Canada data from 2006 were used as a source of patients' median household income for each census dissemination area (DA), an area including approximately 600 households.

\section{Definition of diagnostic interval}

The diagnostic interval was defined as the time from the first CRC-related healthcare visit to the date of CRC diagnosis, as suggested by the Refined Anderson Model of Total Patient Delay within the constraints of the administrative data used for the study [23]. The first
CRC-related healthcare visit was defined as the earliest of three record dates: 1) the most proximal GP visit prior to the first diagnostic imaging test, 2) the most proximal GP visit prior to the first endoscopy, or 3) the first date of GI symptoms (Fig. 1a). For (1) and (2), the date of the most proximal GP visit prior to the respective procedure was assumed to be the date the patient received a referral for the procedure. This assumption was made because in Alberta patients very seldom receive a specialist procedure or see a specialist without a referral. The referral usually comes from a GP. The exception to this is if a patient presents in an urgent state to an emergency department (ED). For this reason, if the first diagnostic imaging or endoscopy was performed in an ED and the patient did not have any prior record of a healthcare visit with GI symptoms then the ED visit was assumed to be the first healthcare contact. A oneyear look-back period from the date of CRC diagnosis was used to identify relevant diagnostic imaging tests and endoscopies based on a sensitivity analysis we conducted that found roughly the same number of tests 12 , 18 , or 24 months prior to colorectal cancer diagnosis. A 6-month look-back period from date of diagnostic imaging or endoscopy was used to identify the GP referral visit - based on the recommendation by the Canadian Association of Gastroenterology [24] for the target maximal wait time from referral to procedure completion in absence of a symptom. To be consistent with the maximum timeframe for GP visits prior to

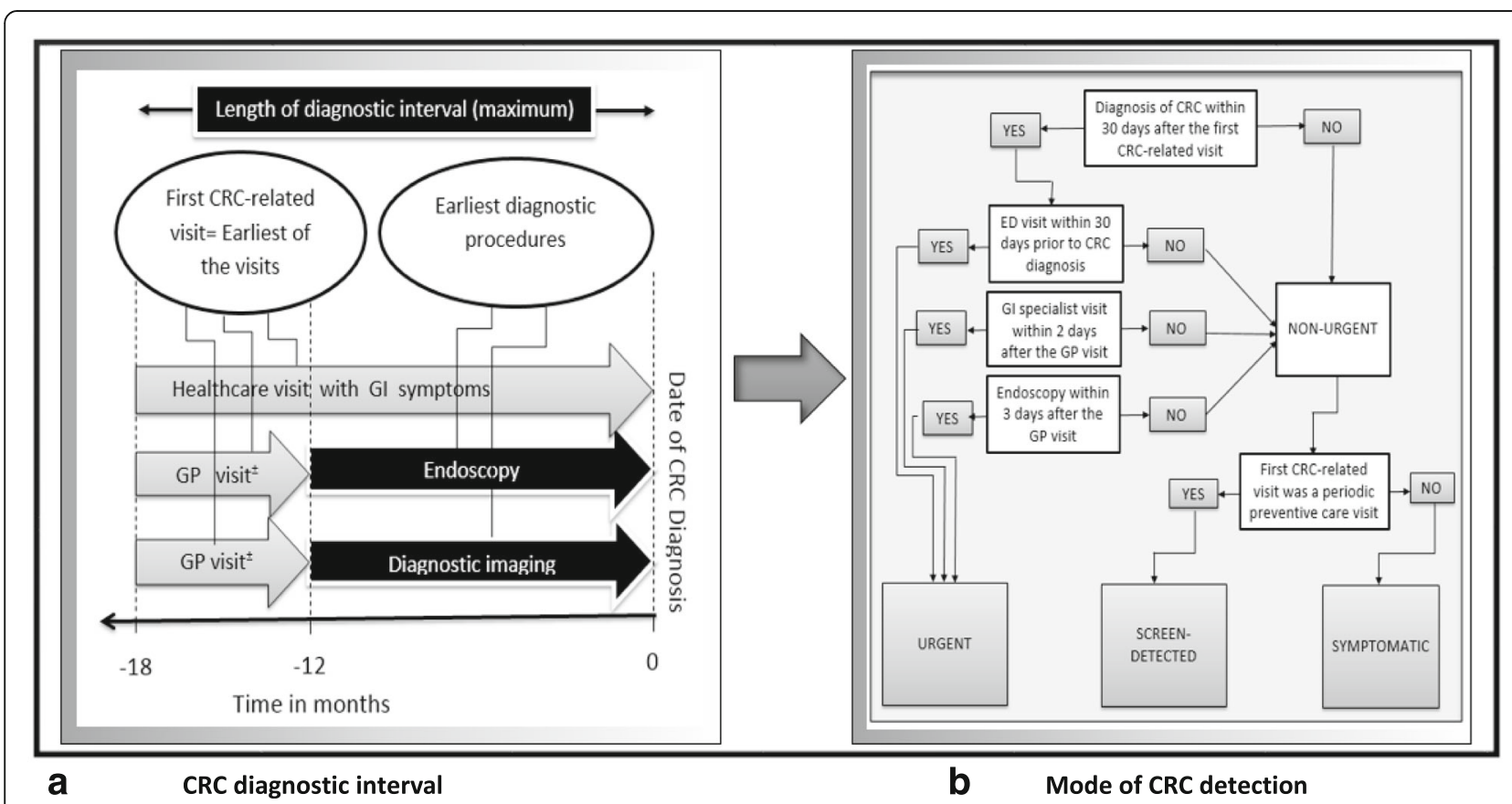

Fig. 1 Illustrative flowchart for a) definition of CRC diagnostic interval and $\mathbf{b}$ ) mode of CRC detection. CRC = Colorectal cancer, Gl = Gastrointestinal, GP= General practitioner, ED = Emergency Department; ${ }^{ \pm} \mathrm{GP}$ visit refers to the most proximal GP visit prior to the first diagnostic imaging test or endoscopy exam 
diagnosis, an 18-month look-back period was used to identify all records with GI-related symptoms.

\section{Definition of mode of CRC detection}

The study patients were classified by their mode of CRC detection: urgent, screen-detected or symptomatic, depicted in Fig. 1b. Presentation was defined as 'urgent' if the diagnostic interval was less than 30 days and the patient had any of the following: 1) an ED visit within the diagnostic interval; 2) a GI specialist visit within 2 days after the GP visit; or 3) an endoscopy performed within 3 days after the GP visit. The rationale for (2) and (3) is based on recommendations from the Canadian Association of Gastroenterology which state that emergency cases should be seen and endoscopy performed within $24 \mathrm{~h}$ following referrals [25]. To allow timing to contact the specialist and preparation for endoscopy, we extended this to 2 and 3 days, respectively. Patients were classified as screen-detected if their first CRC-related healthcare visit was a periodic preventive care visit with a GP, identified by the corresponding procedure code in the Physician Claims data, and they had not been classified as "urgent". At that time, Alberta did not have a formal colorectal cancer screening program, thus the only screening that occurred was opportunistic, typically initiated at periodic preventive care visits. Many Canadians have a preventive care visit, typically once every year, to their family physicians, which is paid by the government's universal healthcare system. Unlike usual primary care, a periodic preventive care visit allows individuals to undertake screening tests recommended based on the patient age, sex, family history, etc. The remaining patients were categorized as "unscreened", and are, by exclusion, non-urgent and likely symptomatic.

\section{Statistical analysis}

The primary outcomes were mode of CRC detection and time to diagnosis. Descriptive statistics were calculated to describe the association of patient demographics, clinical characteristics and healthcare utilization by mode of CRC detection: urgent; screening; or symptomatic. Healthcare utilization factors were categorized based on the frequency distribution of each variable. Chi-square test for categorical variables and JonckheereTerpstra trend test for ordinal variables were used to assess statistical significance.

By definition, the length of the diagnostic interval for urgently diagnosed patients is $\leq 30$ days, therefore, this patient group was excluded from the analyses examining factors related to time to diagnosis. The median and $75^{\text {th }}$ percentile time to diagnosis in days was calculated for each variable of interest. The Kruskal Wallis test was used to assess for differences across medians within each categorical variable. Multivariable quantile regression was used to ascertain the association of demographic, clinical and healthcare utilization factors to the diagnostic interval (days). Quantile regression was used rather than the more typically used ordinary least square regression (OLS) model because the former allows examination of the way factor-effects change with increased time to diagnosis. Unlike OLS, quantile regression makes no distributional assumption about the error term in the model, and it offers considerable model robustness. Furthermore, the interpretation of the estimates is the difference in days associated with each factor relative to the reference group, making it simple to understand. All factors evaluated in the unadjusted analysis were included in the quantile regression except the number of diagnostic imaging tests and unknown provider-type to address issues of co-linearity. Backward elimination method was used to identify the most parsimonious regression model for the $50^{\text {th }}$ and $75^{\text {th }}$ percentiles.

All statistical analyses were performed using statistical software SAS 9.3 (SAS Institute, Cary, NC, USA).

\section{Results}

A total of 11,241 patients were diagnosed in Alberta with colorectal cancer from 2004 to 2010 . There were 1,615 patients excluded from the study for the following reasons: 477 had stage 0 cancer or a cancer histology that was not a CRC solid tumor; 467 had unknown stage; 229 did not have pathologic or microscopic confirmation of disease; 184 had a prior history of CRC or stomach cancer; 134 were diagnosed with another cancer within 6 months prior to the diagnosis of CRC; and 124 had no record of healthcare visits within 1.5 year prior to CRC diagnosis preventing estimation of time to diagnosis. The study cohort included the remaining 9,626 patients.

\section{Patients by mode of CRC detection and related factors}

The median age of patients at diagnosis was 69 years and $57 \%$ were male. Approximately $25 \%$ of patients (2428) presented as urgent, 32\% (3083) were screendetected and $43 \%$ (4115) were non-urgent symptomatic. Table 1 shows the distribution of patient demographic and clinical characteristics by mode of CRC detection. Compared to screen-detected and symptomatic patients, urgent patients were significantly more likely to be older than 80 years, have colon rather than rectal cancer, stage IV tumor and a Charlson comorbidity score of 2 or higher but not have GI-related comorbidities. The screen-detected patients were most likely to be between 60 and 79 years, not have comorbidities, and reside in one of the two major cities, Calgary or Edmonton.

Table 2 shows the relationship between mode of CRC detection and healthcare utilization. Screen-detected patients were most likely to have medium to high 
Table 1 Patient and clinical characteristics by mode of colorectal cancer diagnosis $(n=9626)$

\begin{tabular}{|c|c|c|c|c|}
\hline & $\begin{array}{l}\text { Urgent } \\
(n=2428) \\
n(\%)\end{array}$ & $\begin{array}{l}\text { Screen-detected } \\
(n=3083) \\
n(\%)\end{array}$ & $\begin{array}{l}\text { Symptomatic } \\
(n=4115) \\
n(\%)\end{array}$ & $p$-value ${ }^{a}$ \\
\hline \multicolumn{5}{|l|}{ Patient characteristics } \\
\hline \multicolumn{5}{|l|}{ Age at diagnosis } \\
\hline$<50$ & $228(9)$ & $211(7)$ & $383(9)$ & 0.0429 \\
\hline $50-59$ & $461(19)$ & $519(17)$ & $757(18)$ & \\
\hline $60-69$ & $586(19)$ & $792(26)$ & $1015(25)$ & \\
\hline $70-79$ & $599(25)$ & $1011(33)$ & $1230(30)$ & \\
\hline$\geq 80$ & $554(23)$ & $550(18)$ & $730(18)$ & \\
\hline \multicolumn{5}{|l|}{ Residential zones ${ }^{\mathrm{b}}$} \\
\hline Calgary & $804(33)$ & $1084(35)$ & $1226(30)$ & $<.0001$ \\
\hline Central & $334(14)$ & $357(12)$ & $741(18)$ & \\
\hline Edmonton & $836(34)$ & $1088(35)$ & $1320(32)$ & \\
\hline North & $274(11)$ & $292(9)$ & $463(11)$ & \\
\hline South & $180(7)$ & $262(9)$ & $364(9)$ & \\
\hline \multicolumn{5}{|c|}{ Median annual household income } \\
\hline$<\$ 45389$ & $647(27)$ & $705(23)$ & $993(24)$ & 0.4062 \\
\hline$\$ 45389-\$ 59119$ & $590(24)$ & $681(22)$ & $1074(26)$ & \\
\hline$\$ 59119-\$ 80148$ & $595(25)$ & $789(26)$ & $964(23)$ & \\
\hline$\geq \$ 80148$ & $547(23)$ & $837(27)$ & $962(23)$ & \\
\hline Missing & $49(2)$ & $71(2)$ & $122(3)$ & \\
\hline \multicolumn{5}{|l|}{ Clinical characteristics } \\
\hline \multicolumn{5}{|l|}{ Anatomic site } \\
\hline Colon & $1672(69)$ & $1943(63)$ & $2471(60)$ & $<.0001$ \\
\hline Rectum & $756(31)$ & $1140(37)$ & $1644(40)$ & \\
\hline \multicolumn{5}{|l|}{ Stage at diagnosis } \\
\hline । & $280(12)$ & $781(25)$ & $886(22)$ & $<.0001$ \\
\hline$\|$ & $670(28)$ & $831(27)$ & $1150(28)$ & \\
\hline III & $701(29)$ & $879(29)$ & $1191(29)$ & \\
\hline IV & $777(32)$ & $592(19)$ & $888(22)$ & \\
\hline \multicolumn{5}{|c|}{ Charlson comorbidity score } \\
\hline 0 & $1359(56)$ & $1985(64)$ & $2528(61)$ & 0.0025 \\
\hline 1 & $601(25)$ & $675(22)$ & $893(22)$ & \\
\hline$\geq 2$ & $468(19)$ & $423(14)$ & $694(17)$ & \\
\hline \multicolumn{5}{|c|}{ Gastrointestinal comorbidities } \\
\hline Yes & $544(22)$ & $827(27)$ & $1062(26)$ & 0.0005 \\
\hline No & $1884(78)$ & $2256(73)$ & $3053(74)$ & \\
\hline
\end{tabular}

aJonckheere-Terpstra trend test for ordinal variables (age, income, comorbidity score and stage at diagnosis) and Chi-square test for the other categorical variables were used to compare proportions. ${ }^{b}$ One patient with unknown residential zone was excluded from this calculation

continuity of usual provider care and patients who presented urgently were least likely, $79 \%$ versus $63 \%$, respectively. A small proportion of patients, 5-7\%, had one or more visits with a symptom to a non-GI
Table 2 Healthcare utilization by mode of colorectal cancer diagnosis $(n=9626)$

\begin{tabular}{lllll}
\hline Healthcare Utilization & $\begin{array}{l}\text { Urgent } \\
(n=2428)\end{array}$ & $\begin{array}{l}\text { Screen-detected } \\
(n=3083)\end{array}$ & $\begin{array}{l}\text { Symptomatic } \\
(n=4115)\end{array}$ & $p$-value \\
& $n(\%)$ & $n(\%)$ & \\
& & & & $<.0001$ \\
\hline UPC index & & & \\
$\quad<3$ primary care visits & $276(11)$ & $76(2)$ & $159(4)$ & \\
Low (<50\%) & $620(26)$ & $586(19)$ & $904(22)$ & \\
Medium (50\%-80\%) & $821(34)$ & $1129(37)$ & $1440(35)$ & \\
High (>80\%) & $711(29)$ & $1292(42)$ & $1612(39)$ &
\end{tabular}

Physicians visits with Gl-related symptom

Visits to any physician

$\begin{array}{lllll}0-1 & 1437(59) & 1063(34) & 1138(28) & <.0001 \\ 2 & 631(26) & 1008(33) & 1302(32) & \\ 3 & 218(9) & 452(15) & 767(19) & \\ 4 & 81(3) & 240(8) & 365(9) & \\ \geq 5 & 61(3) & 320(10) & 543(13) & \\ \text { GP visits } & & & & \\ 0-1 & 2093(86) & 1859(60) & 2235(54) & <.0001 \\ 2 & 254(10) & 856(28) & 1230(30) & \\ 3 & 44(2) & 200(6) & 333(8) & \\ \geq 4 & 37(2) & 168(5) & 317(8) & \\ \text { Gl specialist visits } & & & & \\ 0 & 1761(73) & 2106(68) & 2771(67) & <.0001 \\ 1 & 538(22) & 751(24) & 990(24) & \\ \geq 2 & 129(5) & 226(7) & 354(9) & \\ \text { non-Gl specialist visits } & & & & \\ 0 & 2266(93) & 2918(95) & 3868(94) & 0.4904 \\ \geq 1 & 162(7) & 165(5) & 247(6) & \end{array}$

Unknown provider-type visits

$\begin{array}{lllll}0 & 1822(75) & 2411(78) & 2975(72) & <.0001 \\ 1 & 528(22) & 462(15) & 777(19) & \\ \geq 2 & 78(3) & 210(7) & 363(9)\end{array}$

Visits for Gl-related Procedure

Endoscopies

$\begin{array}{lllll}0 & 468(19) & 206(7) & 340(8) & <.0001 \\ 1 & 1657(68) & 2360(77) & 3051(74) & \\ \geq 2 & 303(12) & 517(17) & 724(18)\end{array}$

Diagnostic imaging tests

\begin{tabular}{lllll}
0 & $1092(45)$ & $1551(50)$ & $1893(46)$ & 0.0001 \\
1 & $1002(41)$ & $917(30)$ & $1270(31)$ & \\
2 & $247(10)$ & $381(12)$ & $589(14)$ \\
$\geq 3$ & $87(4)$ & $233(8)$ & $362(9)$ \\
\hline
\end{tabular}

Abbreviations: UPC Usual provider care, GP General practitioner, GI Gastrointestinal, aJonckheere-Terpstra trend test was used to compare proportions

specialist, regardless of mode of CRC detection. Nonurgent symptomatic patients had the highest number of total physician visits prior to diagnosis, with $41 \%$ having 
three or more visits compared to $33 \%$ of screen-detected and $15 \%$ of urgent patients; the increase was primarily due to larger numbers of GP visits with 16, 11 and $4 \%$ of non-urgent symptomatic, screened and urgent patients, respectively, having 3 or more GP visits.

\section{Time to diagnosis}

Table 3 shows the median and $75^{\text {th }}$ percentile time to diagnosis for the non-urgent patients by the demographic, clinical and healthcare utilization factors examined. The overall median and $75^{\text {th }}$ percentile time to diagnosis was 79 and 206 days, respectively. The median time for screen-detected patients was 74 days (interquartile range (IQR) 36-183 days) compared to 84 days (IQR 39-223 days) for symptomatic patients. The oldest patients, those $>80$ years, had the longest diagnostic interval, with a median of 105 days, while patients aged 60-69 years had the shortest median at 69 days. The clinical factor that had the largest relationship to the length of the diagnostic interval was Charlson comorbidity index: those with a score of 2 or more had a median of 147 days compared to 70 days for those with a score of 0 . In general, healthcare utilization factors affected the length of the diagnostic interval much more than patient demographic or clinical factors. The median diagnostic interval for patients with 5 or more CRCsymptom-related visits was significantly longer than the median for those with 0 or 1 CRC-symptom-related visits, 287 days and 38 days, respectively. Similarly, the median diagnostic interval for those with 3 or more CRC-symptom-related visits to a GP was 224 days compared to 56 for those with 0 or 1 visits and 224 days for those with 2 or more CRC-symptom-related visits to a GI specialist compared to a median of 102 days for those with 1 visit to a GI-specialist.

Table 4 presents the results of the quantile regression models at the 50th and $75^{\text {th }}$ percentiles.

The results are qualitatively similar to the unadjusted analysis shown in Table 3 . The demographic and clinical factors with the largest effect were age: median diagnostic interval for oldest patients was 14 days longer than those aged 60-69 years; stage: median diagnostic interval for stage 1 patients was 26 days longer than stage IV patients; Charlson comorbidity score: median diagnostic interval was 32 days longer for those with a score of 2 or more compared to those with a score of 0 .

The number of GP and GI specialist visits for CRC symptoms had the largest impact on the length of the diagnostic interval after adjusting for demographic, clinical and other healthcare utilization factors. Compared to patients with 0-1 GP visit with CRC symptoms, the median diagnostic interval for those with 2 or $\geq 3$ was 44 and 141 days longer, respectively. Similarly, compared to patients who did not have any visits for CRC symptoms to a GI specialist, those who had 1 or $\geq 2$ visits had a median diagnostic interval that was 33 and 108 days longer, respectively. Having any CRC-symptomatic visits to a non-GI specialist, however, was associated with almost 3 months longer median time to diagnosis.

\section{Discussion}

In Alberta, Canada, a province with universal health coverage but without a formal colorectal screening program at the time of the study, $25 \%$ of patients presented as urgent while $20 \%$ had a diagnostic interval over 6 months, representing $45 \%$ of all newly diagnosed CRC patients over a 6 -year period. Tørring et al. has shown that CRC patients with very short or very long diagnostic intervals have higher mortality [10]; efforts are clearly needed to improve the CRC diagnostic care process. Patients most likely to present as urgent were 80 years or older, have colon cancer, stage IV disease, and have multi-morbidities. The demographic and clinical factors that had the greatest impact on the length of the diagnostic interval were age greater than 80 years, stage I disease, and presence of multi-morbidities. Healthcare utilization factors had a much larger effect on the length of the diagnostic interval than clinical factors, however, with each GP or GI specialist visits for symptoms adding more than a month to the median time and a visit to a non-GI specialist for symptoms adding almost 3 months to the median time. Colon cancer is often difficult to diagnose, with non-specific symptoms developing slowly, whose import may be difficult for the patient to recognize, or require investigation or initial referral to a non-GI specialist. Therefore it is possible that many of these patients presented with non-specific findings that led to indirect routes of investigation and referral, before the correct diagnosis was determined. Regardless, these findings suggest that inefficiencies in the healthcare system are the most critical remediable factors to address.

A total of $32 \%$ patients identified as screen-detected via periodic preventive care check-up. Results from a 2009 survey showed that $43 \%$ of Albertan's aged 50-74 years were up-to-date with their CRC screening [26]. This is reasonable given that our study included patients with CRC of all ages, whereas the screening rate is low in younger population. One possible improvement is to introduce a formal CRC screening program to decrease the percentage of patients presenting as urgent [27]. Regular screening addresses the difficulty of non-specific presentations by identifying cancer before symptoms begin. Countries with a national screening program, such as Germany, Italy and Australia, have reported urgent presentation rates ranging from 6 to 19\% [28-30] significantly less than $25 \%$ found in the current study. Building awareness among patients and practitioners in 
Table 3 Median and $75^{\text {th }}$ percentile of time to diagnosis of non-urgent colorectal cancer patients $(n=7198)$

\begin{tabular}{|c|c|c|c|}
\hline \multirow[t]{2}{*}{ Factors } & \multicolumn{3}{|c|}{ Time to diagnosis (days) } \\
\hline & Median & $75^{\text {th }}$ percentile & $p$-value ${ }^{a}$ \\
\hline Overall & 79 & 206 & \\
\hline \multicolumn{4}{|l|}{ Patient and clinical factors } \\
\hline \multicolumn{4}{|l|}{ Age at diagnosis } \\
\hline$<50$ & 81 & 177 & \multirow[t]{5}{*}{$<.0001$} \\
\hline $50-59$ & 74 & 158 & \\
\hline $60-69$ & 69 & 172 & \\
\hline 70-79 & 82 & 223 & \\
\hline$\geq 80$ & 105 & 286 & \\
\hline \multicolumn{4}{|l|}{ Residential zones } \\
\hline Calgary & 78 & 196 & \multirow[t]{5}{*}{0.275} \\
\hline Central & 84 & 227 & \\
\hline Edmonton & 80 & 208 & \\
\hline North & 78 & 223 & \\
\hline South & 74 & 194 & \\
\hline \multicolumn{4}{|c|}{ Median annual household income } \\
\hline$<\$ 45389$ & 80.5 & 234 & \multirow[t]{5}{*}{0.0035} \\
\hline$\$ 45389-\$ 59119$ & 87 & 219 & \\
\hline$\$ 59119-\$ 80148$ & 77 & 196 & \\
\hline$\geq \$ 80148$ & 74 & 177 & \\
\hline Missing & 74 & 278 & \\
\hline \multicolumn{4}{|l|}{ Anatomic site } \\
\hline Colon & 87 & 227 & \multirow[t]{2}{*}{$<.0001$} \\
\hline Rectum & 70 & 171.5 & \\
\hline \multicolumn{4}{|l|}{ Stage at diagnosis } \\
\hline । & 90 & 206 & \multirow[t]{4}{*}{0.0007} \\
\hline$\|$ & 78 & 212 & \\
\hline III & 78 & 211 & \\
\hline IV & 70 & 196.5 & \\
\hline \multicolumn{4}{|l|}{ Charlson comorbidity score } \\
\hline 0 & 70 & 164 & \multirow[t]{3}{*}{$<.0001$} \\
\hline 1 & 88 & 219 & \\
\hline$\geq 2$ & 147 & 322 & \\
\hline \multicolumn{4}{|c|}{ Gastrointestinal comorbidities } \\
\hline Yes & 88 & 195 & \multirow[t]{2}{*}{0.0039} \\
\hline No & 76 & 210 & \\
\hline \multicolumn{4}{|l|}{ Healthcare utilization factors } \\
\hline \multicolumn{4}{|l|}{ UPC index } \\
\hline$<3$ primary care visits & 50 & 112 & \multirow[t]{4}{*}{$<.0001$} \\
\hline Low $(<50 \%)$ & 90 & 231 & \\
\hline Medium (50\%-80\%) & 87 & 223 & \\
\hline High (>80\%) & 73 & 181.5 & \\
\hline
\end{tabular}

Table 3 Median and $75^{\text {th }}$ percentile of time to diagnosis of non-urgent colorectal cancer patients ( $n=7198)$ (Continued)

\begin{tabular}{|c|c|c|c|}
\hline \multicolumn{4}{|l|}{ Visits to any physician } \\
\hline $0-1$ & 38 & 76 & $<.0001$ \\
\hline 2 & 71 & 151 & \\
\hline 3 & 125 & 276 & \\
\hline 4 & 189 & 348 & \\
\hline$\geq 5$ & 287 & 436 & \\
\hline \multicolumn{4}{|l|}{ GP visits } \\
\hline $0-1$ & 56 & 127 & $<.0001$ \\
\hline 2 & 104 & 245 & \\
\hline$\geq 3$ & 226 & 384 & \\
\hline \multicolumn{4}{|l|}{ Gl specialist visits } \\
\hline 0 & 64 & 160 & $<.0001$ \\
\hline 1 & 102 & 245 & \\
\hline$\geq 2$ & 224.5 & 413 & \\
\hline \multicolumn{4}{|l|}{ non-Gl specialist visits } \\
\hline 0 & 76 & 194 & $<.0001$ \\
\hline$\geq 1$ & 201 & 358 & \\
\hline \multicolumn{4}{|l|}{ Endoscopies } \\
\hline 0 & 97 & 271 & $<.0001$ \\
\hline 1 & 71 & 176 & \\
\hline$\geq 2$ & 129 & 285 & \\
\hline \multicolumn{4}{|c|}{ Diagnostic imaging tests } \\
\hline 0 & 61 & 135 & $<.0001$ \\
\hline 1 & 84 & 226 & \\
\hline 2 & 125 & 290 & \\
\hline$\geq 3$ & 183 & 302 & \\
\hline \multicolumn{4}{|l|}{ Method of detection } \\
\hline Screen-detected & 74 & 183 & 0.0002 \\
\hline Symptomatic & 84 & 223 & \\
\hline
\end{tabular}

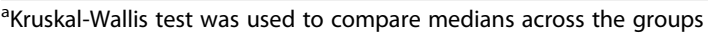

understanding and recognizing the potential seriousness of symptoms is equally important. Educational efforts in combination with routine screening would help to mitigate patient- and practitioner-related factors of late presentation as well as clinical complexities due to nonspecific symptoms [31, 32].

The patient population at highest risk for urgent presentation was those greater than 80 years with multimorbidities. Likewise, these patients were also at highest risk for a long diagnostic interval. Other studies have also found that older patients and those with comorbidities have long diagnostic intervals; this may be due to missed opportunities related to non-specific symptoms, signs, and abnormal tests [33]. Providers may also be distracted from investigating GI symptoms or be hesitant to refer older patients to colonoscopy because of 
Table 4 Quantile regression estimates of the median and $75^{\text {th }}$ percentile of the diagnostic interval for non-urgent colorectal cancer patients $(n=7198)$. The estimate associated with each category is the difference in days when compared to the reference category

\begin{tabular}{|c|c|c|c|c|}
\hline & \multicolumn{2}{|l|}{ Q0.50 } & Q0.75 & \multirow[b]{2}{*}{$P$-value } \\
\hline & Days & $P$-value & Days & \\
\hline Intercept & 7.28 & 0.89 & 15 & 0.08 \\
\hline
\end{tabular}

Age at diagnosis

$<50$
$50-59$
$60-69$
$70-79$
$>80$

Residential Zones

Calgary
Central
Edmonton
North
South
Anatomic site

\section{Rectum}

Stage at diagnosis

।
II
IV

$\begin{array}{llll}25.67 & <.0001 & 42.13 & <.0001 \\ 8.47 & 0.0013 & 14.03 & 0.031 \\ 11 & <.0001 & 23.98 & 0.0001 \\ 0 & . & 0 & .\end{array}$

Charlson Comorbidity Score

$\begin{array}{lllll}0 & 0 & & 0 & \cdot \\ 1 & 5.13 & 0.0371 & 19.21 & 0.0025 \\ \geq 2 & 31.86 & <.0001 & 82.24 & <.0001 \\ \text { Gl comorbidity } & & & & \\ \quad \text { No } & 6.4 & 0.0014 & 29.18 & <.0001 \\ \text { Yes } & 0 & \cdot & 0 & \cdot \\ \text { Usual Provider Continuity } & & & & \\ <3 \text { primary care visits } & 4.52 & 0.35 & 13.54 & 0.39 \\ \text { Low }<=50 \% & 5.47 & 0.07 & 18.34 & 0.0046 \\ \text { Medium 5080\% } & 4.87 & 0.06 & 11.64 & 0.0269 \\ \text { High >80\% } & 0 & \cdot & 0 & \cdot \\ \text { \# of GP visits } & & & & \\ \text { 0-1 } & 0 & \cdot & 0 & \cdot \\ 2 & 44.13 & <.0001 & 91.45 & <.0001 \\ \geq 3 & 140.8 & <.0001 & 201.59 & <.0001 \\ \text { \# of Gl specialist visits } & & & & \\ 0 & 0 & . & 0 & . \\ 1 & 33.46 & <.0001 & 50.68 & <.0001 \\ \geq 2 & 108.54 & <.0001 & 153.56 & <.0001\end{array}$

Table 4 Quantile regression estimates of the median and $75^{\text {th }}$ percentile of the diagnostic interval for non-urgent colorectal cancer patients $(n=7198)$. The estimate associated with each category is the difference in days when compared to the reference category (Continued)

\begin{tabular}{cllll}
\hline $\begin{array}{llll}\text { \# of non-Gl specialist visits } \\
0\end{array}$ & 0 &. & 0 &. \\
$\geq 1$ & 81.55 & $<.0001$ & 101.56 & $<.0001$ \\
\# of endoscopy & & & & \\
0 & 17.44 & $<.0001$ & 52.66 & $<.0001$ \\
1 & 0 &. & 0 &. \\
$\geq 2$ & 36.05 & $<.0001$ & 50.43 & $<.0001$
\end{tabular}

The unconditional percentiles corresponded to diagnostic interval cut-off values are $\mathrm{Q} 0.50=79$ and $\mathrm{Q} 0.75=206$ days

the higher complication rate in this population. Even with a screening program, cases will still present among the old, as will those not detected by screening. Therefore, the health care system must still identify and manage such patients presenting with symptoms.

The median length of the diagnostic interval was 79 days and the $75^{\text {th }}$ percentile was 206 days for screen-detected and symptomatic patients in our study. Two other population-based studies conducted in Canada that included slightly older patient cohorts, diagnosed in years 2001-2005, reported a median diagnostic interval of 44-64 days (depending on year of diagnosis) [12] and 44 days [34]. The two studies used similar methods to ours, however, an important difference is that we excluded urgent patients from the calculation of the diagnostic interval whereas the other two did not. If we include urgent patients, the median diagnostic interval was 55 days in our study with $37 \%$ of all patients diagnosed within 4 weeks, similar to $37.1 \%$ found by Porter et al. [34]. A multicenter US study that included mainly male patients of Veteran Affairs hospitals reported a median diagnostic interval of 91 days for screen-detected and 74 days for symptom-detected CRC [33]. Another observational study in Northern Holland reported a median diagnostic interval of 23.5 weeks (i.e., 165 days) using prospectively collected data [2]. The median diagnostic interval that the present study reported is similar to Fisher et al. [33], but much shorter compared to the other non-Canadian studies [2]. This difference may be partly due to differences in patient populations and study design but could also reflect differences in the healthcare systems.

Among the healthcare utilization factors, the increased number of GI-related healthcare visits for pre-diagnostic work-up had the greatest impact on the length of the diagnostic interval. If the diagnostic interval comprises discrete intervals, the primary care interval and the secondary care interval, as proposed by Olesen et al. [35], this finding is particularly interesting given our methods 
likely underestimate the length of the primary care interval since our start date is likely the date of referral from primary to secondary care. The fact that there were a higher proportion of patients with multiple GP visits than specialist visits suggests that the primary and secondary care intervals overlap in our study population significantly more than the ideal diagnostic care trajectory for a significant proportion of the population. While multiple visits may adversely affect time to diagnosis and the experience of care, our data do not enable us to identify the reasons for multiple visits to physicians: clinical complexity of the disease, misleading symptoms, reasonable watchful waiting, delay in appropriate investigations to confirm diagnosis, or possibly suboptimal professional performance of healthcare providers. A recent review suggests that diagnostic difficulty and the need for investigation of poorly differentiated symptoms in primary care are more likely to be the drivers for multiple visits than poor diagnostic reasoning and suboptimal professional practice [36]. Patient factors, including cancer awareness, beliefs and attitudes before presentation to healthcare system, are also factors contributing to delayed diagnosis [37]. On the contrary, others have found that poor physical examination and misdiagnosis by both primary and hospital physicians might contribute to repeated visits and lengthen time to diagnosis [38]. Increased healthcare utilization may also be due to 1) GP's perception of needing more follow-up visits prior to referring for endoscopy, 2) management during the wait for "non-urgent" specialist consultation and 3) lack of service integration that would allow provision of multiple services at the same patient visit by the same healthcare provider or clinical team [12, 39].

\section{Strengths and limitations}

Several limitations of the current study should be acknowledged. First, there will be a proportion of misclassification of the CRC presentation groups: urgent, screened, and symptomatic. We purposefully used a conservative definition for classifying urgent patients to minimize the chance of overestimation, however, in the absence of procedure codes indicating purpose of a test (i.e., screening or diagnostic) there will be misclassification in the screened and symptomatic groups, specifically, it is likely symptomatic patients who were identified at a preventive care visit are included in the 'screened' group. Second, it is not possible to determine whether patients presented urgently because they ignored early symptoms or because of errors and/or inefficiencies in the healthcare system, Similarly, it is not possible from this study to ascertain the extent of delays between physician appointments and tests due to patient lack of urgency, those due to system inefficiencies, or those due to valid clinical difficulty in diagnosis. In identifying time to diagnosis, we restricted the length of time to a maximum 18 months. This may result in underestimating the length of time to diagnosis for certain individuals given that a few patients may have presented to GP prior to the first contact we identified but is unlikely to have affected the median or $75^{\text {th }}$ percentile, both of which were considerably less than 18 months.

A major strength of the study is that it is populationbased and included all histologically confirmed incident colorectal cancer cases over a 7-year period, minimizing the potential for selection bias. Quantile regression allowed us to estimate the effect factors of interest had directly on the length of the diagnostic interval, with respect to the median and $75^{\text {th }}$ percentile. We also used an innovative definition of urgent presentation by combining multiple events of urgent nature (i.e., time to diagnosis, time and status of ED visit, endoscopy exams and specialist visit).

\section{Implications and areas for further research}

The relationship between timeliness of care and factors associated with the pre-diagnostic healthcare delivery is complex. Actions are needed to reduce the frequency of urgent presentation as well as improve the timeliness of diagnosis. Both of these can be addressed to some extent by implementation of an organized screening program in the eligible general population, however, many cases will continue to present despite the program, and systems must assist these patients too. The effect on resource utilization is critical when developing and implementing an organized screening program, which may increase number of diagnoses [40, 41]. The goal of reducing time to diagnosis can also be achieved by better service integration and coordination of care across service providers [39].

\section{Conclusion}

Major efforts are needed to 1) identify CRC at an early stage when it is treatable, for instance, through an organized screening program and 2) to develop, test, and implement care models that address the healthcare needs of those with multiple morbidities in a timely fashion. A province-wide colorectal screening program has been recently instituted; the current study provides strong baseline data that can be used to evaluate the screening program's effectiveness in decreasing the frequency of urgent presentation and improving timely diagnosis.

\footnotetext{
Abbreviations

CRC: Colorectal cancer; DA: Dissemination area; ED: Emergency department; GI: Gastrointestinal; GP: General practitioner; ICD-O: International classification of disease for oncology; IQR: Interquartile range; OLS: Ordinary least square regression; UPC: Usual provider continuity
} 


\section{Acknowledgements}

This study was supported in part by a grant from the Canadian Institutes of Health Research. The authors would like to thank Truong Minh Pham for his support for data extraction used for this study.

\section{Funding}

This work was supported in part by a grant from the Canadian Institutes of Health Research.

\section{Availability of data and materials}

Due to the requirement to sign confidentiality agreements with the data custodians, we are not at liberty to directly share our datasets. All other materials described in the manuscript, including tables and figures, will be freely available to any scientist wishing to use them for non-commercial purposes, without breaching participant confidentiality.

\section{Authors' contributions}

MW conceived the idea and obtained funding and data for the study. KCS conducted the analysis with oversight from MW and drafted the manuscript. All authors contributed to the study design, interpretation of data analyses, and editing and approval of the final manuscript. JD provided clinical perspective and interpretation. All authors take responsibility for the integrity of the data and data analysis, and read and approved the final manuscript.

\section{Authors' information}

KCS is a biostatistician with 10 years of experience using administrative health data to assess healthcare in Canada. MW is an epidemiologist with a research focus on evaluating quality of cancer care using administrative health data. At the time of the study she was Director of Innovation and Decision Support, Cancer Care Alberta, Alberta Health Services. She is currently Director, Evaluation Sciences Unit, Stanford University. KCS and MW are national leaders in using administrative health data for research. JD is a primary healthcare provider and epidemiologist, and serves on the Canadian Task Force on Preventive Health Care, which undertakes literature synthesis to produce recommendations for primary care providers.

\section{Competing interests}

The authors declare that they have no competing interests.

\section{Consent for publication}

There are no images or other materials relating to individual participants requiring written informed consent for publication.

\section{Ethics approval and consent to participate}

This study was approved by the Health Research Ethics Board of Alberta. Consent to participate was not needed as this was a secondary data analysis based on medical records of the patients available in the population-based administrative health data.

\section{Author details}

${ }^{1}$ Community Health Sciences and O'Brien Institute for Public Health, Cumming School of Medicine, University of Calgary, G 214 HSC, 3330 Hospital Drive NW, Calgary, AB T2N 4N1, Canada. ${ }^{2}$ Departments of Family Medicine and Community Health Sciences, Faculty of Medicine, University of Calgary, Calgary, Canada. ${ }^{3}$ Department of Medicine, Stanford University, Stanford, CA 94305, USA.

\section{Received: 15 October 2015 Accepted: 10 December 2016} Published online: 05 January 2017

\section{References}

1. Canadian Cancer Society. Canadian Cancer Society's Steering Committee on cancer statistics. Toronto: Canadian Cancer Society; 2013.

2. Droste JS T s, Oort FA, van der Hulst RW, Coupe VM, Craanen ME, Meijer GA, Morsink LM, Visser O, van Wanrooij RL, Mulder CJ. Does delay in diagnosing colorectal cancer in symptomatic patients affect tumor stage and survival? a population-based observational study. BMC Cancer. 2010;10:332.

3. Tomlinson C, Wong C, Au H, Schiller D. Factors associated with delays to medical assessment and diagnosis for patients with colorectal cancer. Can Fam Physician. 2012;58(9):e495-501.
4. Schreuders EH, Ruco A, Rabeneck L, Schoen RE, Sung JJY, Young GP, Kuipers EJ. Colorectal cancer screening: a global overview of existing programmes. Gut. 2015;0:13. doi:10.1136/gutjnl. 2014-309086.

5. Major D, Bryant H, Delaney M, Fekete S, Gentile L, Harrison M, Mai V, Nicholson E, Taylor Y. Colorectal cancer screening in Canada: results from the first round of screening for five provincial programs. Curr Oncol. 2013;20(5):252-7. doi:10.3747/co.20.1646.

6. Canadian Partnership Against Cancer. Canadian partnership against cancer's cancer control snapshot on colorectal cancer staging. Toronto: The Canadian Partnership Against Cancer; 2010.

7. Coates AS. Breast cancer: delays, dilemmas, and delusions. Lancet. 1999;353:1112-3.

8. Richards MA. The size of the prize for earlier diagnosis of cancer in England. Br J Cancer. 2009;101 Suppl 2:S125-9.

9. Thomson CS, Forman D. Cancer survival in England and the influence of early diagnosis: what can we learn from recent EUROCARE results? $\mathrm{Br} J$ Cancer. 2009;101 Suppl 2:S102-9.

10. Tørring ML, Frydenberg M, Hansen RP, Olesen F, Hamilton W, Vedsted P. Time to diagnosis and mortality in colorectal cancer: a cohort study in primary care. Br J Cancer. 2011;104:934-40.

11. Porter GA, Inglis KM, Wood LA, Veugelers PJ. Access to care and satisfaction in colorectal cancer patients. World J Surg. 2005;29:1444-51.

12. Singh $H$, De C, Shu E, Fradette K, Latosinsky S, Pitz M, Cheang M, Turner D. Wait times from presentation to treatment for colorectal cancer: a population-based study. Can J Gastroenterol. 2010;24:33-9.

13. Tucker TC, Howe HL, Weir HK. Certification for population-based cancer registries. J Reg Mgmt. 1999;26:24-7.

14. Li X, Hilsden R, Hossain S, Fleming J, Winget M. Validation of administrative data sources for endoscopy utilization in colorectal cancer diagnosis. BMC Health Serv Res. 2012;12:358.

15. Li X, King C, White J, de Gara C, Winget M. Validation of colorectal cancer surgery data from administrative data sources. BMC Med Res Methodol. 2012;12:97.

16. Cunningham $C T$, Cai P, Topps D, Svenson LW, Jetté N, Quan H. Mining rich health data from Canadian physician claims: features and face validity. BMC Res Notes. 2014;7:682.

17. Robertson R, Campbell NC, Smith S, Donnan PT, Sullivan F, Duffy R, et al. Factors influencing time from presentation to treatment of colorectal and breast cancer in urban and rural areas. Br J Cancer. 2004;90(8):1479-85.

18. Hamilton W, Round A, Sharp D, Peters TJ. Clinical features of colorectal cancer before diagnosis: a population-based case-control study. $\mathrm{Br} J$ Cancer. 2005;93:399-405.

19. Korsgaard M, Pedersen L, Sorensen HT, Laurberg S. Reported symptoms, diagnostic delay and stage of colorectal cancer: a population-based study in Denmark. Colorectal Dis. 2006;8(8):688-95.

20. Deyo RA, Cherkin DC, Ciol MA. Adapting a clinical comorbidity index for use with ICD-9-CM administrative databases. J Clin Epidemiol. 1992;45:613-9.

21. Quan H, Sundararajan V, Halfon P, Fong A, Burnand B, Luthi JC, Saunders LD, Beck CA, Feasby TE, Ghali WA. Coding algorithms for defining comorbidities in ICD-9-CM and ICD-10 administrative data. Med Care. 2005;43:1130-9.

22. Knight JC, Dowden JJ, Worrall GJ, Gadag V, Murphy MM. Does higher continuity of family physician care reduce hospitalizations in elderly people with diabetes? Popul Health Manag. 2009;12:81-6.

23. Walter F, Webster A, Scott S, Emery J. The Andersen model of total patient delay: a systematic review of its application in cancer diagnosis. J Health Serv Res Policy. 2012;17:110-8.

24. Leddin D, Armstrong D, Barkun AN, Chen Y, Daniels S, Hollingworth R, Hunt RH, Paterson WG. Access to specialist gastroenterology care in Canada: comparison of wait times and consensus targets. Can J Gastroenterol. 2008;22:161-7.

25. Paterson WG, Depew WT, Paré P, Petrunia D, Switzer C, Veldhuyzen SJ, Zanten V, Daniels S. Canadian consensus on medically acceptable wait times for digestive health care. Can J Gastroenterol. 2006;20:411-23.

26. Canadian Partnership Against Cancer. Canadian partnership against cancer's cancer control snapshot on an update on colorectal cancer screening in Canada. Toronto: The Canadian Partnership Against Cancer; 2012.

27. Hwang $\mathrm{H}$. Emergency presentation of colorectal cancer at a regional hospital: an alarming trend? BC Med J. 2012;54:83-7.

28. Canadian Cancer Society. Canadian Cancer Society's Steering Committee on cancer statistics. Toronto: Cancer Society; 2011.

29. Coco C, Verbo A, Manno A, Mattana C, Covino M, Pedretti G, Petito L, Rizzo G, Picciocchi A. Impact of emergency surgery in the outcome of rectal and left colon carcinoma. World J Surg. 2005;29:1458-64. 
30. Merkel S, Meyer C, Papadopoulos T, Meyer T, Hohenberger W. Urgent surgery in colon carcinoma. Zentralbl Chir. 2007;132:16-25.

31. Mitchell E, Macdonald S, Campbell NC, Weller D, Macleod U. Influences on pre-hospital delay in the diagnosis of colorectal cancer: a systematic review. Br J Cancer. 2008;98(1):60-70.

32. Power E, Simon A, Juszczyk D, Hiom S, Wardle J. Assessing awareness of colorectal cancer symptoms: measure development and results from a population survey in the UK. BMC Cancer. 2011;11:366.

33. Fisher DA, Zullig LL, Grambow SC, Abbott DH, Sandler RS, Fletcher RH El-Serag HB. Determinants of medical system delay in the diagnosis of colorectal cancer within the veteran affairs health system. Dig Dis Sci. 2010;55:1434-41

34. Porter G, Urquhart R, Kendell C, Bu J, McConnell Y, Grunfeld E. Timely access and quality of care in colorectal cancer: a population-based cohort study using administrative data. BMC Res Notes. 2013:6:355.

35. Olesen F, Hansen RP, Vedsted P. Delay in diagnosis: the experience in Denmark. Br J Cancer. 2009;101 Suppl 2:S5-8.

36. Lyratzopoulos G, Wardle J, Rubin G. Rethinking diagnostic delay in cancer: how difficult is the diagnosis? BMJ. 2014;349:g7400. doi:10.1136/bmi.g7400

37. Lyratzopoulos G. Markers and measures of timeliness of cancer diagnosis after symptom onset. Cancer Epidemiol. 2014;38:211-3.

38. Esteva M, Leiva A, Ramos M, Pita-Fernánde S, González-Luján L, Casamitjana M. Factors related with symptom duration until diagnosis and treatment of symptomatic colorectal cancer. BMC Cancer. 2013;13:87. doi:10.1186/14712407-13-87.

39. World Health Organization. Service integration, linkages and triage. In: Operations manual for delivery of HIV prevention, care and treatment at primary health centres in high-prevalence, resource-constrained settings, Edition 1 for field testing and country adaptation. Geneva: World Health Organization; 2008. p. 23. WHO Press.

40. Singh H, Shu E, Demers A, Berstein CN, Griffith J, Fradette K. Trends in time to diagnosis of colon cancer and impact on clinical outcomes. Can J Gastroenterol. 2012;26:877-80.

41. Charters TJ, Strumpf EC, Sewitch MJ. Effectiveness of an organized colorectal cancer screening program on increasing adherence in asymptomatic average-risk Canadians. BMC Health Serv Res. 2013;13:449.

\section{Submit your next manuscript to BioMed Central and we will help you at every step:}

- We accept pre-submission inquiries

- Our selector tool helps you to find the most relevant journal

- We provide round the clock customer support

- Convenient online submission

- Thorough peer review

- Inclusion in PubMed and all major indexing services

- Maximum visibility for your research

Submit your manuscript at www biomedcentral.com/submit

) Biomed Central 